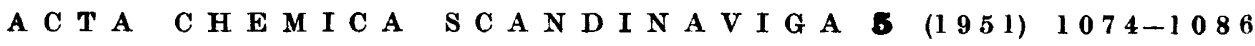

\title{
The Effect of Heat and Urea Treatment on Crystalline Horse Erythrocyte Catalase
}

\author{
H. F. D E U T S C H * \\ Biokemiska avdelningen, Medicinska Nobelinstitutet, Stockholm, Sweden
}

\begin{abstract}
$\mathbf{T}$ The variability in the activity of various crystalline erythrocyte catalases preparations ${ }^{1-5}$ has recently led to the suggestion that this enzyme as usually employed should not be considered a native protein ${ }^{5}$. The preliminary results of denaturation studies also suggested this and it was hoped that further work along these lines might disclose essential differences in various catalase preparations that would be of future value in preparing more uniform material. Such studies as applied to catalase are particularly attractive for one can use a fairly sensitive and rapid activity test for denaturation without having to resort to conditions leading to marked solubility changes as must be so often done with many proteins.

In this investigation the denaturation of several horse erythrocyte catalase preparations by heat and urea treatment showed no evidence of this enzyme being a single chemical entity. Loss of activity as effected by dilution, heating and treatment with urea all suggest the presence of molecules of various labilities.
\end{abstract}

\section{EXPERIMENTAL}

Crystalline horse erythrocyte catalases were prepared by modifications of the method of Bonnichsen ${ }^{3}$ as previously described ${ }^{5}$. Enzyme assays were carried out by the rapid titration method of Bonnichsen, Chance and Theorell ${ }^{6}$ at $18-20^{\circ} \mathrm{C}$ except in one experiment in which the method of von Euler and Josephson ${ }^{7}$ was employed. Various experimental conditions were applied to catalase solutions of such concentration that $0.1 \mathrm{ml}$ of a $1-10$ dilution would give a suitable assay sample. This $1-10$ dilution prior to assay was always carried out with $\mathrm{pH} 6.8, M / 15$ sodium phosphate buffer and served to dilute out the urea, adjust the $\mathrm{pH}$ to the desired assay level and the experimental solution to room temperature, etc. The actual protein concentration of the catalase solutions subjected to various conditions ranged from 0.5 to $1.0 \mathrm{mg}$ per ml depending on the particular enzyme preparations used. These were three in number and showed Kat.F. values of 
61000,117000 and 76000 . They will be referred to as catalase no. 1, 2, and 3 respectively. Catalase no. 2 was the material described previously ${ }^{5}$. The amount of enzyme in any given experiment will always be noted in terms of the first order reaction constant given by the amount of catalase used in the assay.

Absorption spectra were determined by means of a Beckman spectrophotometer. A Zeiss pocket, spectroscope was employed for locating the absorption maxima of the CO hemochromogens which were formed in tests for the presence of free heme resulting from treatment of catalase with urea.

\section{EXPERIMENTAL RESULTS}

\section{Effect of tem perature}

In previous work it was found that catalase showed an initial rapid loss of activity upon dilution ${ }^{5}$. Consequently all studies on the effect of temperature were controlled by running a reaction at either $0^{\circ}$ or $20^{\circ} \mathrm{C}$ employing as nearly as possible the identical conditions of assay as were used with the experimental sample. As will be noted later various catalase preparations showed variable effects as regards the amount of activity lost on dilution.

Little or no denaturation was evidenced at temperatures below $60^{\circ} \mathrm{C}$. It was actually found that the loss of activity resulting from dilution was retarded at temperatures between $20^{\circ}$ and $60^{\circ} \mathrm{C}$. It often appeared that slight decreases in activity in the initial five minutes after dilution tended to be reversed with further incubation and in some cases at $50^{\circ} \mathrm{C}$ the initial activity was found after 30 minutes. The marked loss of activity on dilution was as a rule associated with relatively fresh material. On aging for several weeks in the cold this dilution effect was usually diminished and often small amounts of precipitate had developed in the solutions. The dilution effect was not abolished by use of glass distilled water or of serum albumin solutions as the diluent. If the loss of activity after dilution is due to absorption as has been suggested 8 the effect of temperature above $20^{\circ}$ must be to diminish such absorption. It is also possible that there is an equilibrium between native and denatured catalase which is markedly influenzed by temperature. The effect of temperature on the loss of activity of catalase no. 1 is shown in Fig. 1. The same type of result was observed for preparation no. 2. It is apparent from Fig. 1 that the rate of the denaturation process falls off rapidly with time and that the greater portion of the catalase is stabile at $60^{\circ} \mathrm{C}$ over a 60 minute interval. There appear to be a series of catalase molecules of graded lability as regards their resistance to temperature denaturation. Such an effect will also be noted in the experiments involving urea. The reaction rate of the denaturation process in the initial stage was approximated by extrapolating the denaturation rate (calculated as a first order reaction) for the 5 and 10 
minute period to zero time. No correction was applied for losses in activity as experienced at $0^{\circ}$ and $20^{\circ} \mathrm{C}$ since the results at $50^{\circ} \mathrm{C}$ indicated that for these catalase preparations they were largely abolished at the higher temperatures. It would appear likely that the main destructive process at $60^{\circ}$ and $65^{\circ} \mathrm{C}$ is due primarily to temperature as opposed to the activity loss at $20^{\circ} \mathrm{C}$. Various thermodynamic data were calculated from the extrapolated denaturation reaction rates and are shown in Table 1 .

Table 1. The reaction rates, heat, free energy and entropy change of activation in the heat denaturation of two catalase preparations as studied at $60^{\circ}$ and $65^{\circ} \mathrm{C}$ in $\mathrm{pH} 6.8, \mathrm{M} / 150$ phosphate buffer.

\begin{tabular}{lcc} 
& Catalase no. 1 & Catalase no. 2 \\
\cline { 2 - 3 }$k_{60}$ & $4.9 \times 10^{-4}$ & $6.2 \times 10^{-4}$ \\
$k_{65}$ & $13.5 \times 10^{-4}$ & $17.7 \times 10^{-4}$ \\
$\triangle H^{*}$ & 44540 & 46840 \\
$\triangle F^{\neq}$ & 24800 & 24200 \\
$\triangle S^{\neq}$ & +60 & +68 \\
\hline
\end{tabular}

It is to be emphasized that these data are somewhat qualitative in nature and moreover in view of the results shown in Fig. 1 are data for the more labile portions of the catalase preparations. Catalases no. 1 and 2 while showing some differences in the initial denaturation rate gave quite similar values for the heat of reaction, free energy change and the entropy change of the activation process. It is interesting that these values are of similar magnitude to those reported for the heat denaturation of some other enzymes ${ }^{9}$.

\section{Effect of $\mathrm{pH}$}

The effect of hydrogen ion concentration at various salt concentrations at $60^{\circ} \mathrm{C}$ was also investigated. Since the loss in activity in the first five minutes at this temperature appeared to be most representative of the denaturation process measurements were restricted to this interval. A control sample at $0^{\circ} \mathrm{C}$ was also run. The results are shown in Table 2 and indicate that catalase is most stable at $60^{\circ} \mathrm{C}$ near its isoelectric point ( $\mathrm{pH} 5.5$ ) when the salt concentration is low. It can be seen that there is a considerable loss of activity even at $0^{\circ} \mathrm{C}$. It must be emphasized again that the destruction at $0^{\circ} \mathrm{C}$ may be adsorptive in nature and may bear no counterpart to the activity loss occurring at $60^{\circ} \mathrm{C}$. Results for the control sample were almost identical when carried out at $20^{\circ}$ instead of $0^{\circ} \mathrm{C}$. 
Table 2. Effect of $p H$ on the denaturation of catalase no. 2 at $60^{\circ} \mathrm{C}$.

\begin{tabular}{lllcc}
\hline $\begin{array}{c}\text { Molarity of } \\
\text { buffer }\end{array}$ & $\mathrm{pH}$ & Temperature & $\begin{array}{c}\text { Percent activity } \\
\text { loss in 5 minutes }\end{array}$ \\
\hline & & & $0^{\circ}$ & 30 \\
0.067 & $\mathrm{~A}$ & 4.2 & $60^{\circ}$ & 50 \\
0.067 & $\mathrm{~A}$ & 4.2 & $0^{\circ}$ & 28 \\
0.05 & $\mathrm{~A}$ & 5.6 & $60^{\circ}$ & 31 \\
0.05 & $\mathrm{~A}$ & 5.6 & $0^{\circ}$ & 37 \\
0.5 & $\mathrm{~A}$ & 5.6 & $60^{\circ}$ & 86 \\
0.5 & $\mathrm{~A}$ & 5.6 & $0^{\circ}$ & 29 \\
0.067 & $\mathrm{P}$ & 6.8 & $60^{\circ}$ & 40 \\
0.067 & $\mathrm{P}$ & 6.8 & $0^{\circ}$ & 33 \\
0.067 & $\mathrm{P}$ & 7.8 & $60^{\circ}$ & 70 \\
0.067 & $\mathrm{P}$ & 7.8 & &
\end{tabular}

$\mathbf{A}=$ Sodium acetate

$\mathbf{P}=$ Sodium phosphate

There is usually little difference in the amount of destruction of catalase on dilution in the temperature range from $0^{\circ}$ to $30^{\circ} \mathrm{C}$.

\section{Effect of urea}

Preliminary experiments indicated that at room temperature $5 M$ urea would effect approximately $50 \%$ destruction of the catalase activity in 60 minutes at neutral pH. An initial experiment utilizing this concentration of urea in the presence of $\mathrm{pH} 6.8, M / 150$ sodium phosphate buffer showed a rapid initial decrease in activity followed by a much decreased rate of destruction. The results of this experiment corrected for the loss of a control sample are shown in Fig. 2. It is apparent that a relatively rapid destruction is effected within the first hour with this catalase preparation (no. 1). The destruction showed no evidence of being a first order reaction over the prolonged period. Further experiments with urea utilized an incubation period of an hour or less.

Data for the reaction of catalase no. 1 at various urea concentrations in $\mathrm{pH} 6.8, M / 150$ phosphate buffer at $20^{\circ} \mathrm{C}$ were plotted to give Fig. 3. The loss of activity is very rapid initially and then shows only relatively slight decreases. This is reminiscent of the effect of temperature as shown in Fig. 1. The influence of increased concentrations of urea is readily seen from Fig. 3. In another experiment at $18^{\circ} \mathrm{C}$ and in the same buffer the denaturation reaction rate was determined for various urea concentrations. As in the case of the 


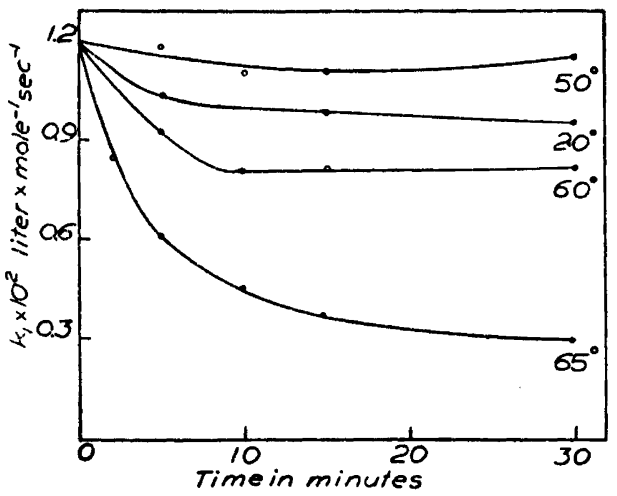

Fig. 1. Effect of temperature on loss of catalase activity in $p H$ 6.8, M/150 phosphate buffer.

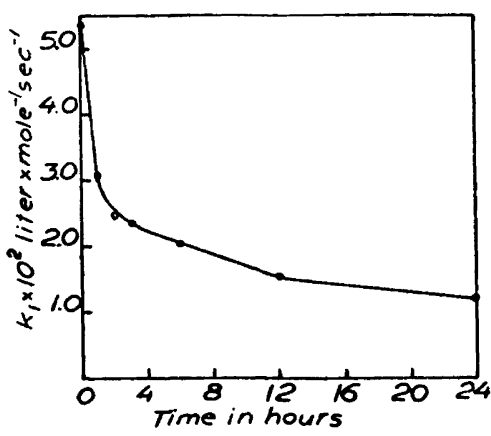

Fig. 2. Loss in catalase activity in $5 M$ urea, pH $6.8 \mathrm{M} / 150$ phosphate buffer at $20^{\circ} \mathrm{C}$. The catalase assay in this experiment was carried out by the method of von Euler and Josephson (7).

experiments involving temperature destruction only the results for the 5 and 10 minute incubation periods were utilized. The denaturation rate, calculated as a first order reaction, was extrapolated to zero time and data for various urea concentrations are given in Table 3. These data as well as those to be given shortly for the effect of temperature variation on the urea denaturation of catalase are subjected to the same limitations as pointed out earlier.

Table 3. The reaction rate for the denaturation of catalase no. 1 at various urea concentrations and in $p H 6.8, M / 150$ phosphate buffer at $20^{\circ} \mathrm{C}$.

\begin{tabular}{cc}
\hline Urea molarity & $k_{1}$ denaturation \\
\hline 3 & $1.0 \times 10^{-4}$ \\
5 & $3.2 \times 10^{-4}$ \\
6 & $11.0 \times 10^{-4}$ \\
7 & $17.5 \times 10^{-4}$ \\
7.5 & $25 \times 10^{-4}$ \\
\hline
\end{tabular}

Lauffer ${ }^{10}$ has calculated that the velocity constant for urea denaturation at constant temperature is proportional to a power of the urea concentration. The slope of a plot of the above type of data is indicative of the number of urea molecules bound. The data of Table 3 was plotted in such a manner to give Fig. 4 which, while not showing a completely satisfactory straight line 


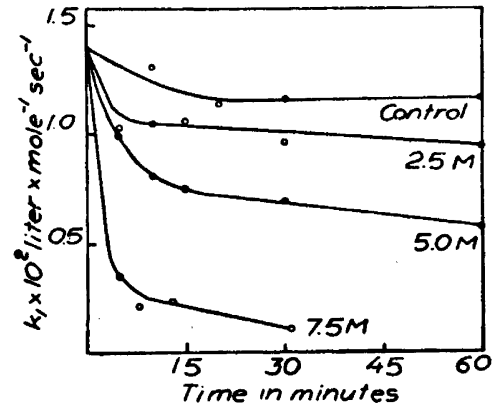

Fig. 3. Effect of various concentrations of urea on catalase in $p H 6.8 \mathrm{M} / 150$ phos. phate buffer at $20^{\circ} \mathrm{C}$.

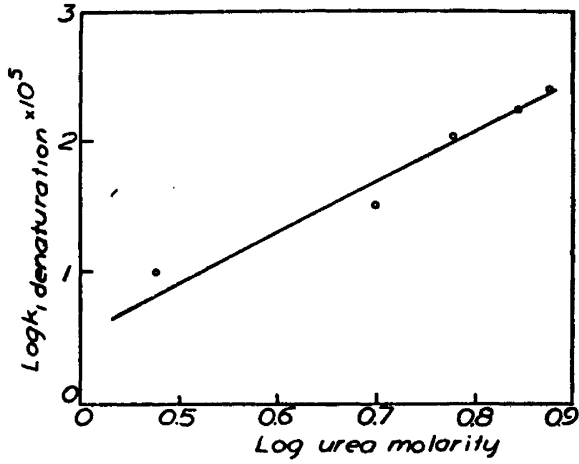

Fig. 4. The logarithm of the extrapolated denaturation velocity constant for catalase plotted against the logarithm of the urea concentration.

relationship, indicates that nearly four urea molecules are bound per molecule of protein at $20^{\circ} \mathrm{C}$. This is less than that found by Lauffer ${ }^{10}$ for tobacco mosaic virus but somewhat higher than that found by Mihályi ${ }^{11}$ for bovine fibrinogen.

\section{Effect of salt concentration}

The denaturation of catalase in $5 M$ urea at $20^{\circ} \mathrm{C}$ was also studied in the presence of $\mathrm{pH} 6.8$ phosphate from 0.05 to $1.0 \mathrm{M}$. A very slight increase in the denaturation rate was seen at the higher phosphate concentrations. This is in keeping with the effect of acetate on the heat denaturation at $\mathrm{pH} 5.6$.

\section{Reversal of urea denaturation}

Attempts were made to determine whether losses in catalase activity were reversible upon dilution of the urea. Catalase preparations that had undergone from $50-75 \%$ decrease in activity as a result of treatment with 5 and $7.5 M$ urea were diluted $1-1.0$ with $M / 150, \mathrm{pH} 6.8$ phosphate buffer. After periods up to 18 hours after dilution there sometimes appeared to be a slight recovery of activity as compared with the appropriate controls. This recovery never exceeded $10 \%$ of the initial activity and was usually of such a low magnitude as to fall within the realm of experimental error. The results, however, were always in the direction of increased activity.

Catalase samples treated with $9 M$ urea at $20^{\circ}$ show precipitation upon dilution with the phosphate buffer. Catalase solutions more concentrated than 
$1 \mathrm{mg}$ protein per $\mathrm{ml}$ when treated with $5 \mathrm{M}$ urea at $30-40^{\circ} \mathrm{C}$ often also show precipitation upon dilution.

\section{Effect of temperature}

The denaturation of catalase no. 1 in $5 M$ urea and in $\mathrm{pH} 6.8, M / 150$ phosphate buffer was also studied over a temperature range of $0-50^{\circ} \mathrm{C}$. The denaturation reaction rate was determined by extrapolation of the data obtained in the initial stages of the reaction to zero time as previously described. The various thermodynamic data calculated for catalase under the above conditions are given in Table 4.

Table 4. The reaction rates, heat, free energy and entropy change of activation in the denaturation of catalase no. 1 in $5 M$ urea in $p H 6.8, M / 150$ phosphate buffer at various temperatures.

\begin{tabular}{ccccc}
\hline Temperature & $k_{1}$ denaturation & $\Delta H^{\ddagger}$ & $\Delta H^{\neq}$ & $\Delta S^{\neq}$ \\
\hline 0 & $2.1 \times 10^{-4}$ & & & \\
10 & $2.94 \times 10^{-4}$ & 3300 & 21200 & -63 \\
20 & $3.5 \times 10^{-4}$ & 3900 & 21000 & -58 \\
30 & $5.3 \times 10^{-4}$ & 6800 & 22200 & -51 \\
40 & $16.7 \times 10^{-4}$ & 20400 & 22300 & -6 \\
50 & $120 \times 10^{-4}$ & 91400 & 21900 & +215 \\
\hline
\end{tabular}

The heat of activation of the denaturation process is lowered considerably by the presence of urea between $0^{\circ}$ and $40^{\circ} \mathrm{C}$. A marked rise, however, is noted at $40^{\circ} \mathrm{C}$ and this becomes quite large at $50^{\circ} \mathrm{C}$. The urea-protein complex formation is exothermic and the number of molecules of urea molecules bound should decrease with a rise in temperature. Correspondingly the entropy and heat of activation will rise with increasing temperature and the latter value should approach that found in the absence of urea at the higher temperatures. Actually the heat of activation at $50^{\circ} \mathrm{C}$ in $5 \mathrm{M}$ urea is much higher than that found at $60-65^{\circ} \mathrm{C}$ in the absence of urea. The reason for this, while not readily apparent, may be the result of the destruction of molecules of quite different chemical reactivities in the two cases. This is quite possible for in the case of $5 M$ urea approximately $96 \%$ of the activity is destroyed within 5 minutes as $50^{\circ} \mathrm{C}$ whereas the data of Fig. 1 show that at $60^{\circ}$ and $65^{\circ} \mathrm{C}$ only $20-50 \%$ of the catalase activity is lost in the absence of urea in the same time interval. The data from $0^{\circ}$ through $30^{\circ} \mathrm{C}$ gives a fairly good Arrhenius plot but deviates markedly above the latter temperature. At these higher temperature 
conditions the more stable catalase molecules are undergoing destruction and it would appear that the heat of activation for the denaturation of these molecules is relatively high. In these studies it must be remembered that we are dealing with data representing an average for molecules of various labilities. This is especially true for the higher temperature, where an increasingly greater percentage of the more stable catalase molecules are undergoing destruction. The free energy change of the activation remains relatively constant since the heat and the entropy of activation show a compensating rise.

\section{Effect of $\mathrm{pH}$}

These studies were carried out on catalase no. 3. This material showed a very high activity loss on dilution but the use of adequate controls allows for the demonstration of the effect of $\mathrm{pH}$ on the denaturation process by urea. The experiments utilized $5 M$ urea at a temperature of $20^{\circ} \mathrm{C}$. Acetate and phosphate buffers of low concentration $(0.035-0.05 M)$ were employed to regulate the $\mathrm{pH}$. The results are shown in Table 5 and indicate that as in the case of the temperature denaturation (Table 2) there is a greater stability of catalase at $\mathrm{pH} \mathrm{5.6.} \mathrm{At} \mathrm{this} \mathrm{hydrogen} \mathrm{ion} \mathrm{concentration} \mathrm{the} \mathrm{loss} \mathrm{of} \mathrm{activity} \mathrm{on}$ dilution of the control is also less and the presence of the $5 M$ urea actually appears to retard this loss in the initial stages. This may suggest that the dilution loss and the urea denaturation do not concern identical molecules. It is difficult to accept the very large dilution loss of catalase no. 3 as one due to absorption on the walls of the container. No change in extinction coefficient at $405 \mathrm{~m} \mu$ is seen on dilution under the same conditions that give huge activity decreases. Apparently a variable portion of the catalase preparations contain molecules of extreme lability. After standing for two weeks at $2-5^{\circ} \mathrm{C}$ catalase no. 3 showed a marked decrease in the dilution effect and a decrease in activity. This was synchronous with the appearance of a small amount of precipitate in the sample.

\section{Spectrophotometric studies}

Since the denaturation kinetics suggested that the catalase preparations employed were made up of a series of molecules of varying reactivitios it appeared desirable to attempt to determine whether the enzyme showed any changes in spectral properties after treatment with urea. It was found that urea caused a decrease in the absorption at the 405 and $620 \mathrm{~m} \mu$ maxima but had little or no effect on the protein absorption $(280 \mathrm{~m} \mu)$. Catalase solutions of the same concentration or lower than those used in the previously discussed 


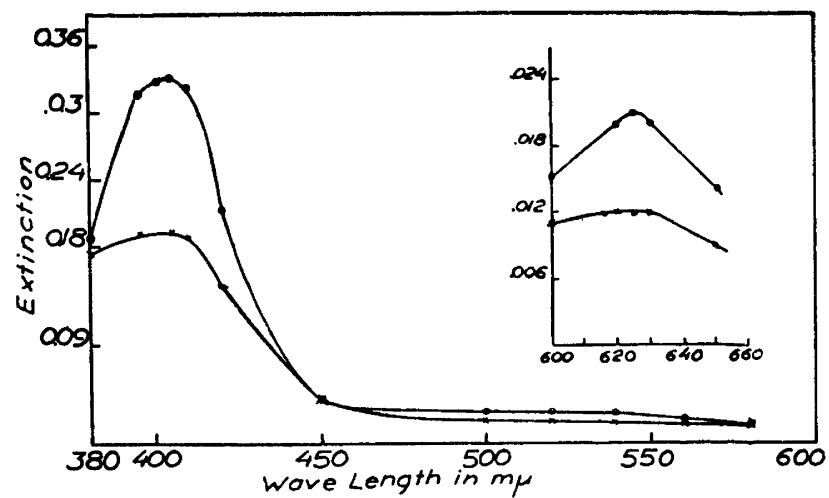

Fig..5. Absorption spectrum of catalase in $5 M$ urea in pH 6.8, M/150 phosphate buffer at $20^{\circ} \mathrm{C}$.

$-0-0-0=$ at zero time

$-x-x-x-x=$ after 60 minutes incubation.

denaturation studies were employed because definite turbidities developed in $5 M$ urea with a consequent marked increase in light absorption in the lower spectral region (below $350 \mathrm{~m} \mu$ ) at higher protein concentrations. The absorption spectrum of catalase no. 1 after 60 minutes in $5 M$ urea is shown in Fig. 5. It is to be noted that the relative decreases in extinctions at 405 and $625 \mathrm{~m} \mu$ are identical. The rate of the extinction change with time was also recorded and is plotted to give Fig. 6. No change in absorption at $280 \mathrm{~m} \mu$ was apparent. The rate of decrease in the extinction at $405 \mathrm{~m} \mu$ is qualitatively similar to the activity decrease for this preparation under the same conditions as shown by inspection of Fig. 3. After incubation for 60 minutes with $5 M$ urea the decrease in extinction coefficient was $43 \%$. This is to be compared to the activity decrease of $60 \%$. Usually the decrease in activity was somewhat greater than the change in extinction coefficient. Different catalase preparations, however, show varying reactivities as regards the rate of spectral change in $5 M$ urea. Whereas catalase no. 1 showed a $43 \%$ decrease in the absorption at $405 \mathrm{mu}$ after 60 minutes, catalase no. 2 showed only a $25 \%$ decrease. Preparation no. 3 was similar to no. 2 in this respect.

A single experiment on the effect of $5 M$ urea on the absorption spectrum of human hemoglobin and of horse myoglobin was also carried out. Both of these proteins showed a decrease (approximately 15-20\%) in the Soret absorption maxima. Contrary to the results for horse erythrocyte catalase they showed an increase in the absorption from $600-650 \mathrm{~m} \mu$ on treatment with urea. The decrease in the absorption maxima in the Soret region for the above 
Fig. 6. Rate of change of absorption at 280 and $405 \mathrm{m \mu}$ by catalase in $5 \mathrm{M}$ urea in $\mathrm{pH}$ $6.8, M / 150$ phosphate buffer at $20^{\circ} \mathrm{C}$.

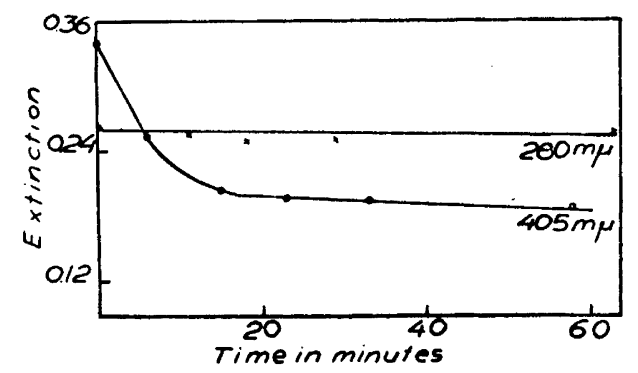

two hemoproteins appeared to be almost instantaneous in contrast to the catalase reaction. No further studies of the effect of urea on hemoglobin or myoglobin were carried out.

The decrease in the absorption maxima of the catalase at 405 and $625 \mathrm{~m} \mu$ suggested that heme was being liberated as a result of the urea treatment. Proof of this was obtained in the following way. A catalase solution was treated with $5 M$ urea at $30^{\circ} \mathrm{C}$ for approximately one hour. The sample was then adjusted to $\mathrm{pH} 9$ and dithionite added. The latter will reduce ferri heme to the ferro compound but is unable to effect this reduction of the iron in catalase. Following this $\mathrm{CO}$ was passed into the solution to form the CO ferroheme complex which at $\mathrm{pH} 9$ should be rapidly converted into the hemochromogen. The controls for this experiment were a sample of pure heme and a solution of catalase to which urea was added immediately before treatment with CO. Inspection of these solutions with a hand spectroscope showed the presence of free heme in the catalase sample treated with urea. Attempts to put this test on a quantitative basis so that the decrease in absorption coefficient at 405 and $625 \mathrm{~m} \mu$ could be compared directly with the rate of appearance of free heme in the solution were unsuccessful.

\section{DISCUSSION}

With few exceptions protein denaturation and biological inactivation have been reported to be first-order reaction rate processes. In our experience catalase does not obey such a reaction although it is possible that various species of catalase molecules may show this property individually. The nature of the heat and urea denaturation processes for horse erythrocyte catalase indicate the presence of molecules of widely varying reactivities in crystalline and supposedly pure enzyme preparations.

Catalase shows a strong positive temperature coefficient for the urea denaturation process as has been reported for such proteins as fibrinogen ${ }^{\mathbf{1 1}, 12}$, 
a carboxyhemoglobin ${ }^{13}$ and chorionic gonadetrophin ${ }^{14}$. Although $\alpha \beta$-lactoglobulin ${ }^{15}$ and egg albumin ${ }^{16,17}$ show a negative temperature coefficient in this respect at high urea concentrations, Clark ${ }^{18}$ has found a positive coefficient at lower urea concentrations for the latter protein. He has suggested that apparent negative temperature coefficients may be the result of secondary solubilizing reactions at the higher urea concentrations ${ }^{10}$. In this respect the use of an enzyme such as catalase avoids such considerations of solubility for one can utilize activity as a reaction guide. It is quite likely in the case of catalase that activity losses indicate earlier protein modifications than those represented by loss of solubility for when the latter occurred in this study almost all enzyme activity had disappeared. Furthermore, marked loss of activity in most cases was not synonomous with loss of solubility under the conditions utilized in this work.

Catalase was found to be most stable to both heat and urea denaturation near its isoelectric point. Apparently low concentrations of salt are to be preferred under any conditions which tend to give denaturation. The application of these two conditions to the more or less rigorous fractionation steps used in the preparation of catalase may possibly result in recovery of more uniform material.

A point particularly of future interest in the urea denaturation of catalase should be to determine whether this reagent causes a molecular dissociation. Horse hemoglobin dissociates to half molecules in the presence of $6.6 \mathrm{M}$ urea ${ }^{20-22}$. Various other proteins ${ }^{23-27}$ also show this type of reaction but substances such as egg albumin ${ }^{20}$, serum albumin ${ }^{28}$, gliadin ${ }^{29}$, zein ${ }^{24}$, pepsin ${ }^{22}$ and several hemoglobins other than the equine form show no such change.

If horse erythrocyte catalase like its hemoglobin counterpart and the latter proteins undergoes a molecular dissociation in strong urea solutions it will be interesting to determine the effect of this on enzyme activity. Horse hemoglobin which has been split in this manner shows differences in oxygen equilibrium as compared to the intact molecule ${ }^{30}$. It was noted that catalase activity losses in urea were higher than the decreases in absorption at 405 and $625 \mathrm{~m} \mu$. It is possible that along with the removal of heme from the protein by the action of urea there is likewise a dissociation into two or more molecules and that in this form the catalase shows a diminished or loss of activity.

It would appear that such investigations would best await the development of fractionation methods giving more uniform catalase preparations of Kat.F. at least 120000 . Consistent evidence exists for the presence of material of this activity in erythrocytes. However, it is possible (but not likely) that erythrocyte catalases exist in nature as a series of molecules of varying activity and that such high activity material represents the more active portions of the enzyme. 
Table 5. The effect of $p H$ on the loss of activity of catalase no. 3 in $5 \mathrm{M}$ urea at $20^{\circ} \mathrm{C}$.

\begin{tabular}{|c|c|c|c|c|}
\hline \multirow[t]{2}{*}{ pH } & \multirow{2}{*}{$\begin{array}{c}\text { Minutes } \\
\text { incubation }\end{array}$} & \multicolumn{2}{|c|}{$k_{1} \times 10^{2}$} & \multirow{2}{*}{$\frac{k_{1 \text { urea }} \times 10^{2}}{k_{1 \text { control }}}$} \\
\hline & & Control & Urea & \\
\hline \multirow{5}{*}{4.0} & 0 & 2.93 & 2.93 & \\
\hline & 5 & 1.12 & 0.98 & 88 \\
\hline & 10 & 1.01 & 0.79 & 78 \\
\hline & 15 & 1.02 & 0.68 & 67 \\
\hline & 0 & 2.93 & 2.93 & \\
\hline \multirow{3}{*}{5.6} & 5 & 1.41 & 1.46 & 104 \\
\hline & 10 & 1.31 & 1.02 & 78 \\
\hline & 15 & 1.31 & 0.96 & 73 \\
\hline \multirow{5}{*}{6.8} & $\mathbf{0}$ & 2.93 & 2.93 & \\
\hline & 5 & 1.13 & 1.01 & 89 \\
\hline & 10 & 1.10 & 0.91 & 83 \\
\hline & 15 & 1.12 & 0.85 & 76 \\
\hline & 0 & 2.93 & 2.93 & \\
\hline \multirow{3}{*}{7.8} & 5 & 1.46 & 1.53 & 105 \\
\hline & 10 & 1.46 & 0.65 & 45 \\
\hline & 15 & 1.42 & 0.55 & 39 \\
\hline
\end{tabular}

\section{SUMMARY}

Various crystalline horse erythrocyte catalase preparations appear to consist of a series of molecules of various reactivities. The enzymatic inactivation of this enzyme by heat and urea does not follow a first order reaction. The enzyme is most stable near its isoelectric point and in the presence of urea is more readily inactivated by the presence of high salt concentrations. Urea causes a decrease in the absorption maxima at 405 and $625 \mathrm{~m} \mu$ that appears to be synonomous with the release of heme from the molecule.

The author wishes to acknowledge his indebtedness to Professor Hugo Theorell for his interest and helpful suggestions during the course of this investigation.

\section{BIBLIOGRAPHY}

1. Laskowski, M., and Sumner, J. B. Science 94 (1941) 615.

2. Agner, K. Arkiv. Kemi, Mineral. Geol. B 17 (1943) no. 9.

3. Bonnichsen, R. K. Arch. Biochem. 12 (1947) 83.

4. Herbert, D., and Pinsent, J. Biochem. J. 43 (1948) 203.

5. Deutsch, H. F. Acta Chem. Scand. 5 (1951). 
6. Bonnichsen, R. K., Chance, B., and Theorell, H. Acta Chem. Scand. 1 (1947) 685.

7. von Euler, H., and Josephson, K. Ann. 452 (1927) 158.

8. Unpublished data from this laboratory.

9. Neurath, H., Greenstein, J. P., Putnamn, F. W., and Erikson, J. O. Chem. Rev. 34 (1944) 157.

10. Lauffer, M. A. J. Am. Chem. Soc. 65 (1943) 1793.

11. Mihalyi, E. Acta Chem. Scand. 4 (1950) 317.

12. Diebold, W., and Jühling, K. Biochem. Z. 296 (1938) 389.

13. Drabkin, D. L. Proc. Soc. Exp. Biol. Med. 41 (1939) 225.

14. Bischoff, F. J. Biol. Chem. 158 (1945) 29.

15. Jacobsen, C. F., and Christensen, K. K. Nature 161 (1948) 30.

16. Hopkins, F. G. Nature 126 (1930) 328, 383.

17. Ramsden, W. Nature 126 (1930) 685.

18. Clark, J. H. J. Gen. Physiol. 27 (1943-1944) 101.

19. Clark, J. H. J. Gen. Physiol. 28 (1944-1945) 539.

20. Burk, N. F., and Greenberg, D. M. J. Biol. Chem. 87 (1930) 197.

21. Wu, H., and Yang, E. F. Chinese J. Physiol. 6 (1932) 51.

22. Steinhardt, J. J. Biol. Chem. 123 (1938) 543.

23. Weber, H. H., and Stöver, R. Biochem. Z. 259 (1933) 269.

24. Burk, N. F. J. Biol. Chem. 120 (1937) 63.

25. Stanley, W. M., and Lauffer, M. A. Science 89 (1939) 345.

26. Gralén, N. Biochem. J. 33 (1939) 1342.

27. Burk, N. F. J. Biol. Chem. 133 (1940) 511.

28. Burk, N. F. J. Biol. Chem. 98 (1932) 353.

29. Burk, N. F. J. Biol. Chem. 124 (1938) 49.

30. Wyman, J. Jr. Advances in Protein Chem. 4 (1948) 410.

Received April 27, 1951. 